\title{
Design and Practice of Mooncake Packaging for Sustainable Development
}

\author{
Minglei Jiang \\ Neusoft Institute of Guangdong \\ Foshan, China 528225
}

\begin{abstract}
The mooncake has nearly a thousand years history, its kinds have been gradually increased as time goes, and the packaging design of it is also changed with the continuous development of the society, but unfortunately, as the mooncake coat, mooncake packaging, in recent years has not been satisfactory. The mooncake image, in people's minds, is beautiful and warm, we need to explore a continuous development method that suitable for the packing of it so as to inherit and develop this kind of traditional food, moon cake, under the state of creating a conservation oriented society and sustainable development in the country plan requirements.
\end{abstract}

Keywords-mooncake packing; material; continuous development; technology

\section{INTRODUCTION}

When the Mid-autumn festival is celebrated, the mooncake is indispensable, which is not only a kind of traditional food, but also embodys people's expectation to the harmonious and happy life, and also their focus on reuniting the family members.

\section{THE PRESENT STATUS OF MOOKCAKE PACKAGING}

In recent years, the packaging and design of the mooncake has goes to the malformed development path under driving the business interests and profit: Because of "face of ego" relating to the gift, the mooncake packaging, which should be classified as the accessory, began to sing the leading role; while the mooncake, which is supposed to be the protagonist, has become as the titular "accessories". For the ordinary mooncake, the value ratio between it and its packaging is to go halves, and for high-grade mooncake, it can reach 2:1 or even higher. Even more incredible is that there are high-grade tobacco, tea and other so-called gifts in these huge boxes. These not only distorts the warmth and good image of mooncake in people's minds, but also against the country's call to create a conservation oriented society and don't meet the requirements of sustainable development plans, and also do not conducive to the inheritance and development of the traditional food.

\section{A. At the Visual and Structural Aspects, the Mooncake Packaging Ignores the Psychological Feelings of Consumers}

In the minds of Chinese people, the affinity of mooncakes has narrowed the distance between people. But in the lavish wrapping of the mooncake, the human touch of mooncakes seems to have been faded. These packaging not only has a complex making process which can be compared with the one of the crafts, but mostly in color with red or yellow as the main colors. Some boxes are made of gold and silver, and even the boxes are lined with yellow silk. In Chinese's mind, yellow and silver colors are the symbol of noble status and also arouse the admiration to people. When most of the mooncake gift boxes are presented in front of the consumer in this style, the resplendent visual effect will make consumers feel psychologically sense of distance.

In addition, in the packaging structure, under the layer upon layer package, (generally, the innermost layer is the single packing by the plastic film as its packaging material, and then put it into the paper box or the steel box, and finally put it into the outer packing box), when the consumer opens the outer packing box, it is difficult to know the true face of the mooncake; the consumer is difficult to judge the quality of mooncake due to it's difficult to see the mooncake itself, which will generate a sense of insecurity, also affect consumer s' trust to it.

\section{B. The Continuous Development Requirements Have Been Ignored During the Design of Mooncake Packaging}

The continuous development is a key topic that has relations with the human's future. We'll find that the packaging of mooncake at present doesn't meet this requirement when we value the current mooncake packaging according to the continuous development requirements:

At first, in recent years, the main trend of the packaging and design of mooncake is luxury and waste, and this trend that focus on luxury packaging and pays no attention to frugality and recycle grow more and more, which warp the packaging meaning.

Secondly, the most direct result brought by the luxury packaging of mooncake is to increase too much unnecessary rubbish. And the final processing way is landfill treatment, to pile up and embed the city life garbage will take a lot of valuable land resource, in addition, there are many hard degradation materials or toxic, which will pollute the soil and environment.

Third, the luxury packaging boxes are basically manually done, a series of materials like the production workshop, plates, dehydration, paper and printing ink and so on can not 
talk about hygiene, which also increases the risk of food contamination. The wooden packing box contain a greater crisis: professionals have pointed out that at present all kinds of wooden boxes on the market usually use density board, multilayer board and fiber board adopted in the decoration and furniture production, the adhesives used in the production of these materials are urea formaldehyde, it is easily absorbed by food, which will easily lead to eye, nose, throat and upper respiratory tract disease after eating, ". These are all second pollution; the toxic gas sewage pollution in the production of these materials is more serious.

\section{To Explore the Future Market Ignored During the Design of Mooncake Packaging}

The fierce fights on mooncake get all "firepower" together to the present market, and don't pay attention to cultivate the "reserve force"-it means the youth, who will be the main body of consumption, the one who win the good feeling of them will win the future market, too. More and more open economy society makes this generation have no trouble on sealing of information, have no much memory on traditional culture, and has no profound historical burden." With the growing up of them, they will become as the main body of consumption gradually, thus their knowledge on the mooncake will decide the business status of the mooncake industry.

\section{To Set the Brand Image Ignored During the Packaging of Mooncake Design}

Brand is the key factor to get success in competition. No mooncake supplier at current market has set up its own brand image via the packaging of it, which style is in a mess, and there is not too much difference on the packaging among all mooncake manufacturers.

\section{ThOUGHTS ON THE SUSTAINABLE DEVELOPMENT OF MOONCAKE PACKAGING}

\section{A. The Sustainable Development of Mooncake Culture}

In Chinese's mind, the mooncake is a kind of festival food which is always filled with a human warm, and then the emotion factors contained by the mooncake should be embodied in the design of its packaging. The mooncake packaging should make the consumers feel the same warm brought by the mooncake itself via its special design; especially in the current era of market economy, the product is variety, but the feeling is gradually weakened, the mooncake packing design taking into account the consumer's emotional needs is a successful packaging design, because once the emotional factors in packaging design resonates with the consumers, then the effect will undoubtedly be lasting and positive.

\section{B. The Packaging Design Should Fin Its Inspiration from the Traditional Culture}

Our country has a very long packing history. Different people, different areas have developed many unique packing styles according to their own habits and location. Such as: pack the meat with lotus leaf, pack the wine with the cucurbit and reasonable packing styles like these. Beyond that, there many styles of packaging, liking to use the popular "Bajiao bag" to pack the medical material, cakes and scattered goods like these; The full Doufang structure together with the small square red paper, and be matched with a simple yellow color can show a festive and auspicious feeling. Thus, to suitable learn from the traditional culture will provide a chance to change the current packaging design of the mooncake.

Along with the rapid rise of the commodities boom and the packaging industry, the packaging waste also grows fast, and some packaging materials such as plastic is difficult to recycle, partly because the recovery and management of local government is not good, in addition to the people's awareness of environmental protection is still relatively backward, they often discarded the waste at will, which will cause serious environmental pollution, the excessive mooncakes exacerbated the process of environmental pollution and ecological damage. In order to protect the environment for human survival and achieve the goal of sustainable development, the best way is to use green packaging materials. This kind of material is in line with the principle of "4R1D": packaging material usage reduction (Reduce), can be used repeatedly (Reuse), regeneration (Recycle) and renewable energy (Recover) of packaging material waste, waste can be natural degradation (Degradable). As a common member of the packaging family, the mooncake packaging should also actively respond to this trend of development.

\section{Sustainable DeVElopMENT DESIGN SCHEME OF MOONCAKE PACKAGING}

\section{A. Visual Design Scheme}

It is as shown in "Fig. 1"

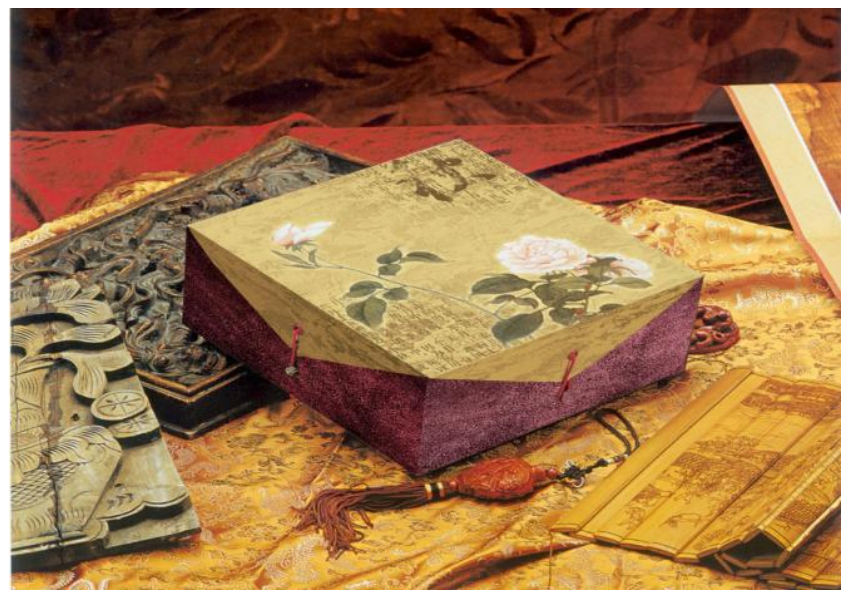

Fig. 1. Visual design scheme of mooncake packaging.

\section{B. Material Design Scheme}

In ancient China, people began to use the existing natural packaging material to package the goods, these materials are nothing more than cotton, wool, hemp, bamboo, wood, grass and so on, they are derived from natural, which not only has adequate resources, meets the requirements of the green 
packaging materials, but also has a distinctive local characteristics and local breath. If you make good use of it, you will get twice the result with half the effort. In the author's scheme, the main material is pure cotton. The material conforms to the green packaging "4R1D" principle, which adopts the waste energy regeneration and waste can be natural degradation principle.

\section{Technology Design Scheme}
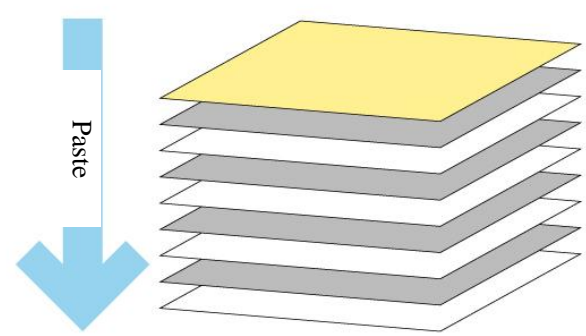

All kinds of material are combined with the traditional paste, then make it flat and dry

original single color cotton paste made of rice and bran

The surface layer, and can use plant dye, or the use of other materials, such as silk, silk, silk, hemp, etc.
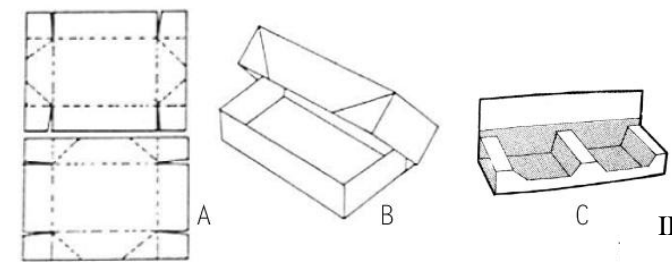

The upper composite material is fabricated according to the structural design, and the packing case and the inner structure are made

A- according to the design, cut out the unfolded box shape, $\mathrm{B}$ - box shape, $\mathrm{C}$ - internal structure

Fig. 2. Technology design scheme of mooncake packaging .

Select from traditional handicrafts and combine with the material requirements of mooncake packaging design, the cotton cloth, as a packaging material, should increase the hardness so as to make it as the packing box, the author selects the process with reference to the production process of traditional China shoes sole - making technology of strong cloth soles. Past the pure cotton cloth layer by layer via the rice chaff and boiled paste to meet the hardness and toughness (e.g., Fig. 2).

Box surface printing, you can use vegetable dyes to print on the surface cotton. According to the mooncake properties, the one with high-grade materials and its box patterns can choose batik, tie dye and traditional technology like them, and the surface material is optional for silk, silk, linen, denim etc.

\section{CONCLUSION}

As a delicious food carrying the traditional culture and Chinese nostalgia, the packing design should have a new look in the new era. The packaging design of the mooncake should take the times as its background, take the public sentiment as its base to arouse the emotional resonance of the consumer via the unique design of the era aesthetics and environmental protection concept so that the delicious food, mooncake, and its related traditional culture can be carried on.

\section{REFERENCES}

[1] Judith Miller.Miller's 20th Century Design[M].London:Octopus, Renke He. Edit 3 of Industrial Design Hitory[M]. Beijing: High Grade Education Press,2004,153-160

[2] Research and Analysis on Green Packaging Design, Guoxin Yang Ping Yang, Packaging Project. 3 Edit in 2006

[3] Xiao Xu Analysis on Ecological Environment from the Mooncake Packaging[J] Today Publish, 10 Edit in 2004

[4] Li Zheng. Analysis on Market Competition from the Mooncake Packaging[J]. Shanghai Packing. 2 Edit in 2006

[5] Zhiyong Guo. Positioning Study on Packing Design on Gift [D]. Master degree candidate of Nanchang University in 2006

[6] Huiqin Huang, Dandan Dai, Xingfu Xiong. Discussion on Mooncake Packing [J]. Packing Project. 5 Edit in 2004. 